\title{
IMPROVEMENT OF MICROWAVE ABSORBANCE OF POLYMER COMPOSITES OF W-TYPE HEXAFERRITE POWDERS BY ATTACHMENT OF FREQUENCY SELECTIVE SURFACE
}

\begin{abstract}
This work investigates the effect of a frequency selective surface (FSS) composed of a regular array of square loop elements on the absorption properties of grounded ferrite composites. Polymer matrix composites of CoZnW hexaferrite powders having small magnetic loss were used as the substrate material. Computational tools were used to model the interaction between electromagnetic waves and materials and determine the reflection coefficient. Reflection loss and bandwidth were greatly improved by attaching an FSS with controlled electrical resistance $(R)$ onto the grounded ferrite composites. For the FSS with $R=800 \Omega$, the minimum reflection loss decreased to $-25 \mathrm{~dB}$ at $10 \mathrm{GHz}$ and the bandwidth was broadened to $7.5-12.5 \mathrm{GHz}$ with respect to $-10 \mathrm{~dB}$ reflection loss.

Keywords: Frequency selective surface, Microwave absorbers, Hexaferrite powders, Polymer composites
\end{abstract}

\section{Introduction}

In resonant absorbers with a quarter wavelength, zero reflection can be obtained by impedance matching at the surface of the absorbing layer, which requires an appropriate combination of magnetic permeability and dielectric permittivity at a given thickness and frequency of interest [1,2]. Based on transmission line theory, the impedance matching can be obtained when the real part of the input impedance is equal to free space impedance $(=377 \Omega)$ and the imaginary part is zero. To date, many types of ferromagnetic metal and ferrite particles in tile or composite forms have been demonstrated which satisfy the impedance matching condition, and these have been proposed as radar absorbing materials (RAM) in various applications [3-7]. In some cases, however, the impedance matching cannot be obtained because the intrinsic material parameters are insufficient, or they limit the matching thickness required for specific applications.

In order to address these issues and improve RAM performance, new design techniques have been proposed, including frequency selective surfaces (FSS). FSS are planar periodic structures of identical patches or apertures of conducting elements, and are generally employed in front of a grounded dielectric slab (substrate) to synthesize high-impedance absorbing surfaces $[8,9]$. The behavior of an FSS can be modeled by an equivalent effective resistor-inductor-capacitor $(R L C)$ circuit $[10,11]$. In such an $R L C$ circuit, the shunted admittance can be represented as a function of $R, L$, and $C$, which depend on the electrical resistance and geometry of the FSS $[10,12,13]$. Combining an FSS and traditional RAM can produce new impedance matching, and, thereby, result in improvements in microwave absorbance and bandwidth, especially for unmatched RAM.

This study investigates the effect of an FSS composed of a regular array of square loop (SL) elements on the absorption properties of grounded ferrite composites. Polymer matrix composites of CoZnW hexaferrite powders having small magnetic loss were used as the substrate material. It was verified by both HFSS simulation and circuit theory that microwave absorbing properties (reflection loss, bandwidth) were greatly improved by attaching an FSS with controlled surface resistance onto the grounded ferrite composites.

\section{Experimental and simulation}

CoZnW hexaferrite powders with a composition of BaCo$\mathrm{ZnFe}_{16} \mathrm{O}_{27}$ were prepared by solid state reaction process. Silicone rubber was used as a matrix material and mixed with the ferrite powders whose content was $80 \%$ by weight. The mixture was molded in a coaxial die with $3 \mathrm{~mm}$ in inner-diameter and $7 \mathrm{~mm}$ in outer-diameter. The complex permeability and permittivity were determined from the measured reflected and transmitted scattering parameters, using a network analyzer.

Computational tools (HFSS 13.0) were used to model the interaction between electromagnetic waves and the materials in the structures shown in Fig. 1. The absorber structure is composed of a square loop FSS (SL-FSS) on the top layer and a perfect electric conductor (PEC) on the bottom layer, separated by the ferrite composite substrate. An electromagnetic wave (with wave

\footnotetext{
* DEPARTMENT OF ADVANCED MATERIALS ENGINEERING, CHUNGBUK NATIONAL UNIVERSITY, CHEONGJU 361-763, KOREA

* Corresponding author: sskim@chungbuk.ac.kr
} 
vector $\mathrm{k}$ ) is incident normal to the composite structure with the electric field (E) and magnetic field $(\mathrm{H})$ polarized as shown in Fig. 1(a). The dimension of the unit cell and SL-FSS is given in Fig. 1(b). The resistance of the SL element was varied in the range of $400-800 \Omega$ by varying electrical conductivity at a fixed film thickness $\mathrm{t}=0.02 \mathrm{~mm}$.
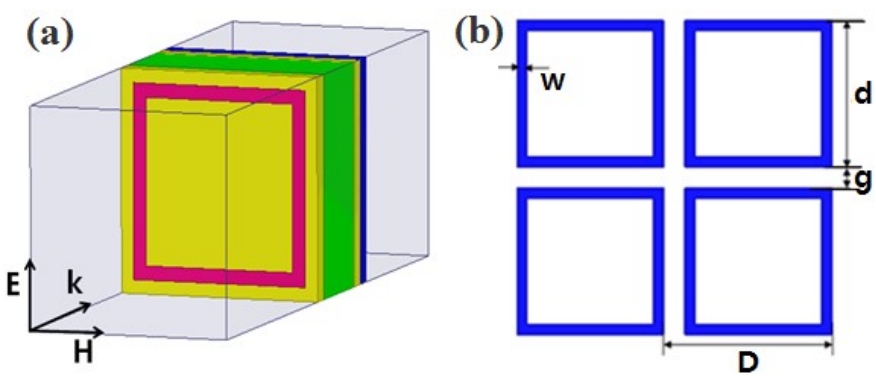

Fig. 1. Schematic description of (a) SL-FSS on grounded ferrite composite and $(b)$ its dimension $(\mathrm{D}=10 \mathrm{~mm}, \mathrm{~d}=8.75 \mathrm{~mm}, \mathrm{w}=0.625 \mathrm{~mm}$, $\mathrm{g}=0.625 \mathrm{~mm})$

\section{Results and discussion}

Fig. 2 shows the complex permeability $\left(\mu_{r}=\mu_{r}{ }^{\prime}-j \mu_{r}{ }^{\prime \prime}\right)$ and permittivity $\left(\varepsilon_{r}=\varepsilon_{r}{ }^{\prime}-j \varepsilon_{r}{ }^{\prime \prime}\right)$ determined in the rubber composites containing CoZnW hexaferrite powders. The real part of the permeability $\left(\mu_{r}{ }^{\prime}\right)$ decreases continuously from 2.3 at $0.05 \mathrm{GHz}$ to 1.0 at $18 \mathrm{GHz}$, and a broad magnetic loss $\left(\mu_{r}{ }^{\prime \prime}\right)$ spectrum is observed up to $18 \mathrm{GHz}$. The peak value of $\mu_{r}{ }^{\prime \prime}$ is approximately 0.6 at $5 \mathrm{GHz}$. The dielectric constant is $\varepsilon_{r}{ }^{\prime} \approx 6.4$, and dielectric loss is negligible $\left(\varepsilon_{r}{ }^{\prime \prime} \approx 0\right)$.
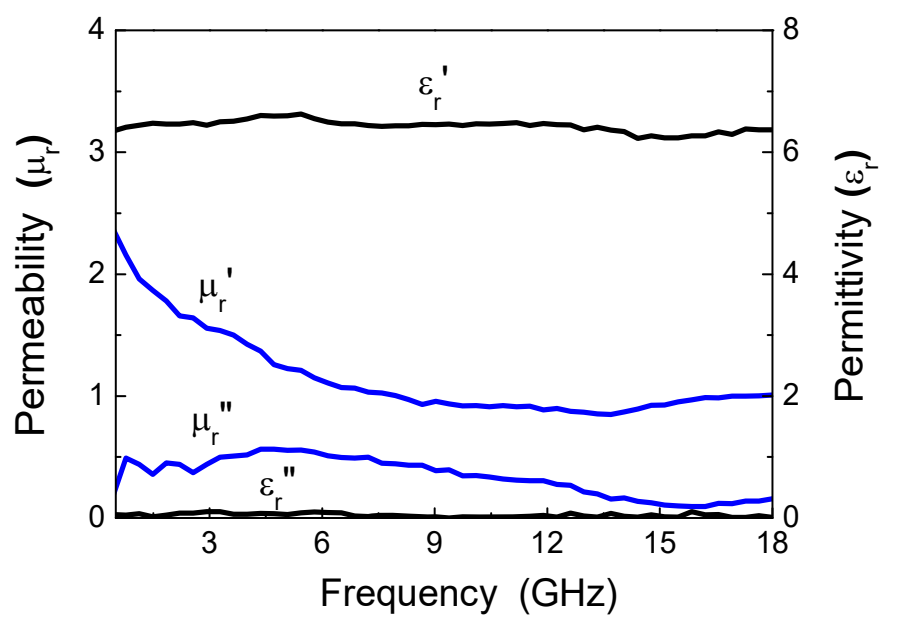

Fig. 2. Complex permeability and permittivity of rubber composite containing CoZnW hexaferrite powders

Fig. 3 shows the reflection loss of the ferrite composite grounded by a PEC with various layer thicknesses. The absorption frequency band moves to lower frequencies with increasing layer thickness. However, the reflection loss is not less than $-20 \mathrm{~dB}$ at all frequencies and layer thickness.

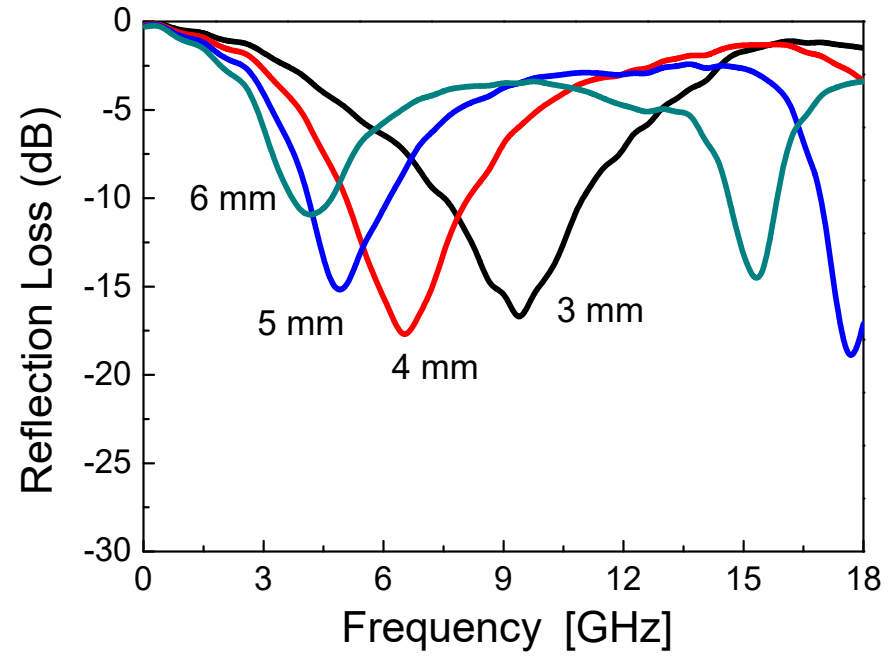

Fig. 3. Reflection loss determined in the grounded ferrite composites with various layer thicknesses

The SL-FSS was attached on the top layer of the grounded ferrite composite, whose thickness was $2.9 \mathrm{~mm}$, corresponding to the quarter wavelength thickness at $10 \mathrm{GHz}$. Fig. 4 shows the simulation results of reflection loss for the composite structure with dimensions of $D=10 \mathrm{~mm}, d=8.75 \mathrm{~mm}, w=0.625 \mathrm{~mm}$, $g=0.625 \mathrm{~mm}$, shown in Fig. 1. It is evident that the reflection loss decreases and the bandwidth broadens as the electrical resistance of SL-FSS $(R)$ increases. In the case of $R=800 \Omega$, the minimum reflection loss decreases to $-25 \mathrm{~dB}$ at $10 \mathrm{GHz}$ and the bandwidth is broadened to $7.5-12.5 \mathrm{GHz}$ with respect to $-10 \mathrm{~dB}$ reflection loss, which is significantly improved as compared with the grounded ferrite composite without FSS.

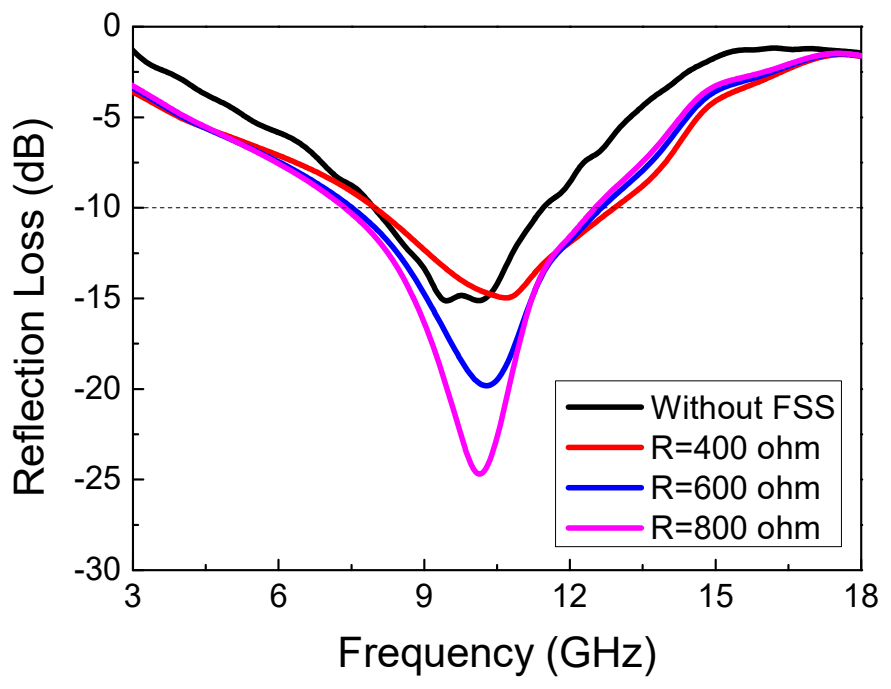

Fig. 4. Reflection loss determined in the composite structure, implemented with $S L-F S S$ on a grounded ferrite composite (thickness = $2.9 \mathrm{~mm}$ ), with increasing $S L-F S S$ resistance $(R)$

The reflection loss is determined by the input admittance of the high impedance surface $(Y)$, which is a parallel circuit of the input admittance of the grounded substrate $\left(Y_{d}\right)$ and the complex admittance of the FSS ( $\left.Y_{F S S}\right)$, expressed as, 


$$
\begin{gathered}
Y=Y_{d}+Y_{F S S}=Y_{0}[Y(R e)+j Y(\operatorname{Im})] \\
Y_{d}=-j Y_{0} \sqrt{\frac{\varepsilon_{r}}{\mu_{r}}} \tanh ^{-1}\left(\frac{2 \pi d}{\lambda} \sqrt{\mu_{r} \varepsilon_{r}}\right) \\
=Y_{0}\left[Y_{d}(R e)+j Y_{d}(\operatorname{Im})\right] \\
Y_{F S S}=\frac{1}{\left[R+j\left(\omega L-\frac{1}{\omega C}\right)\right]} \\
=Y_{0}\left[Y_{F S S}(R e)+j Y_{F S S}(\operatorname{Im})\right]
\end{gathered}
$$

where, $Y(R e)$ and $Y(I m)$ are the real and imaginary parts of the input admittance of the high impedance surface normalized by free space admittance $\left(Y_{0}=1 / 377 \Omega^{-1}\right), \lambda$ is the wavelength in free space, $d$ is the substrate thickness, and $R, L, C$ are the resistive, inductive, and capacitive components of $Y_{F S S}$, respectively. Perfect absorption can be obtained at the impedance matching condition, given by $Y(R e)=1$ and $Y(\operatorname{Im})=0$.

The normalized input admittance of the grounded substrate $\left(Y_{d} / Y_{0}=Y_{d}(R e)+j Y_{d}(\operatorname{Im})\right)$ for a thickness $d=2.9 \mathrm{~mm}$ is shown in Fig. 5. Short-circuit resonance is found at $10 \mathrm{GHz}$ where quarter wavelength thickness is $2.9 \mathrm{~mm}$. With respect to the frequency of $Y_{d}(\operatorname{Im})=0$, the circuit is inductive $\left(Y_{d}(\operatorname{Im}) \leq 0\right)$ at lower frequencies and capacitive $\left(Y_{d}(\operatorname{Im}) \geq 0\right)$ at higher frequencies. The real part $Y_{d}(R e)$ is smaller than 1 at almost all frequencies, which is due to the small value of magnetic losses of the ferrite composite, especially in the region of 9-16 GHz. Due to this admittance spectrum, the microwave absorbance is not so great, as seen in Fig. 4.

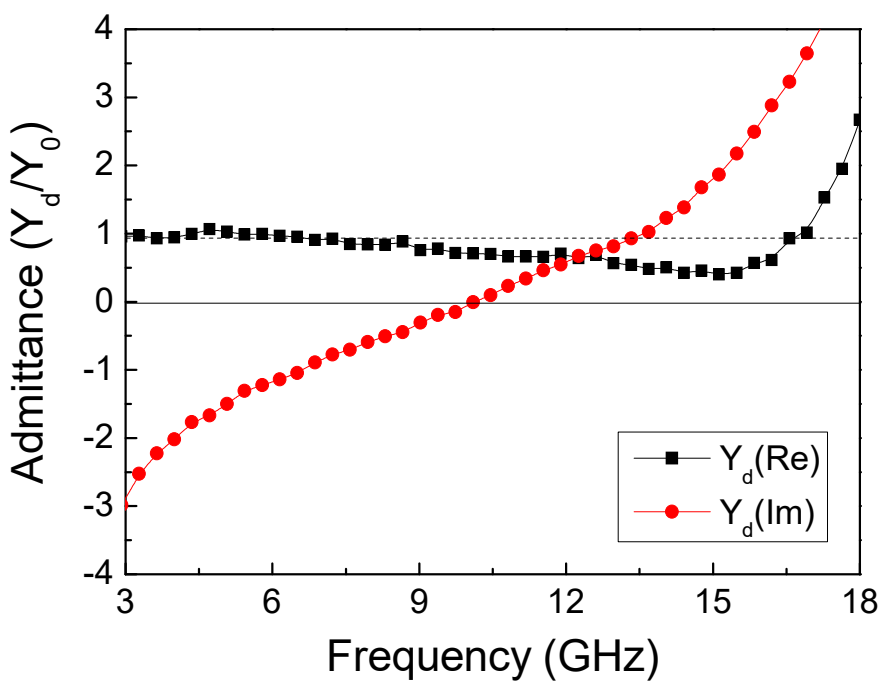

Fig. 5. Normalized real and imaginary parts of input admittance of the grounded ferrite substrate $\left(Y_{d} / Y_{0}=Y_{d}(R e)+j Y_{d}(I m)\right)$

Fig. 6 shows the normalized complex admittance of $S L$ $F S S\left(Y_{F S S} / Y_{0}=Y_{F S S}(R e)+j Y_{F S S}(\operatorname{Im})\right)$ calculated from the $L$ and $C$ values determined by equivalent circuit model $[12,13]$. For the $S L-F S S$ with dimensions shown in Fig. 1(b), $L$ and $C$ values were calculated to be $3.4 \times 10^{-9} \mathrm{H}$ and $72.3 \times 10^{-15} \mathrm{~F}$, respectively, resulting in a $L C$ resonance at $10 \mathrm{GHz}$. With respect to this resonance frequency, the circuit is capacitive $\left.\left(Y_{F S S}(\operatorname{Im})\right) \geq 0\right)$ at lower frequencies and inductive $\left.Y_{F S S}(\operatorname{Im}) \leq 0\right)$ at high frequencies. The broad spectrum of the real part $\left(Y_{F S S}(R e)\right)$ is observed with a peak value at resonance frequency, due to the relatively large surface resistance of the $S L-F S S(R)$. Both the real and imaginary values of $S L-F S S$ admittance decrease with increasing $R$, as shown in Fig. 6.
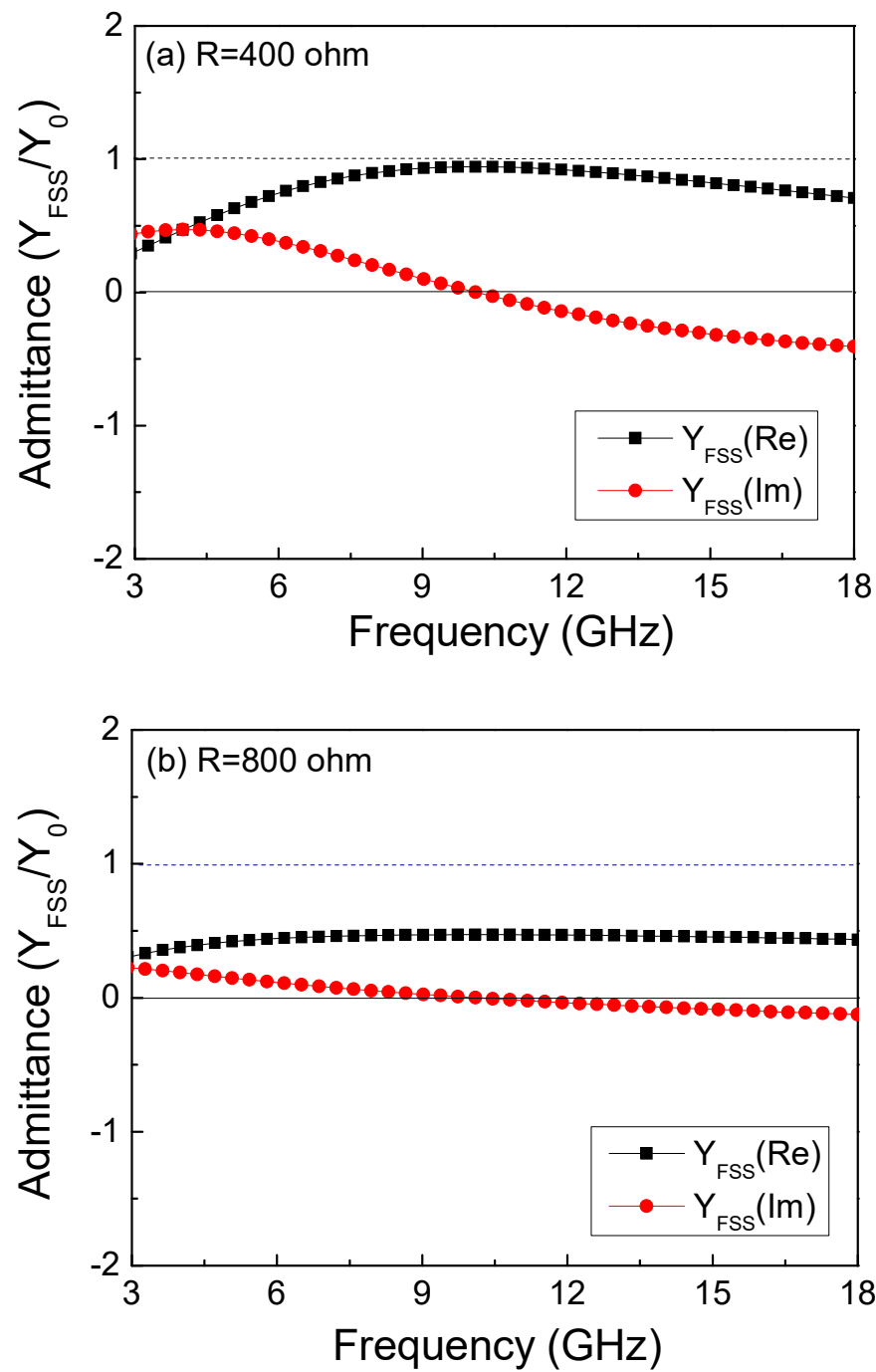

Fig. 6. Normalized real and imaginary parts of complex admittance of $S L-F S S$ with varying varying $S L-F S S$ resistance: (a) $R=400 \Omega$, (b) $R$ $=800 \Omega$

The parallel circuit combination of $Y_{d}$ and $Y_{F S S}$ approach the impedance matching condition $(Y(R e)=1$ and $Y(\operatorname{Im})=0)$ around $10 \mathrm{GHz}$, as shown in Fig. 7. The cancellation effect of inductive and capacitive reactance between $Y_{d}(\mathrm{Im})$ and $Y_{F S S}(\mathrm{Im})$ leads to total admittance $Y(\operatorname{Im}) \approx 0$ in a wider frequency range, resulting in broader bandwidth. The summing effect of $Y_{d}(R e)$ and $Y_{F S S}(R e)$ makes the total real admittance $Y(R e) \approx 1$ around the resonance frequency, especially for large $S L-F S S$ resistance $(R=800 \Omega)$, as shown in Fig. 7(b), resulting in a more reduced reflection loss. 

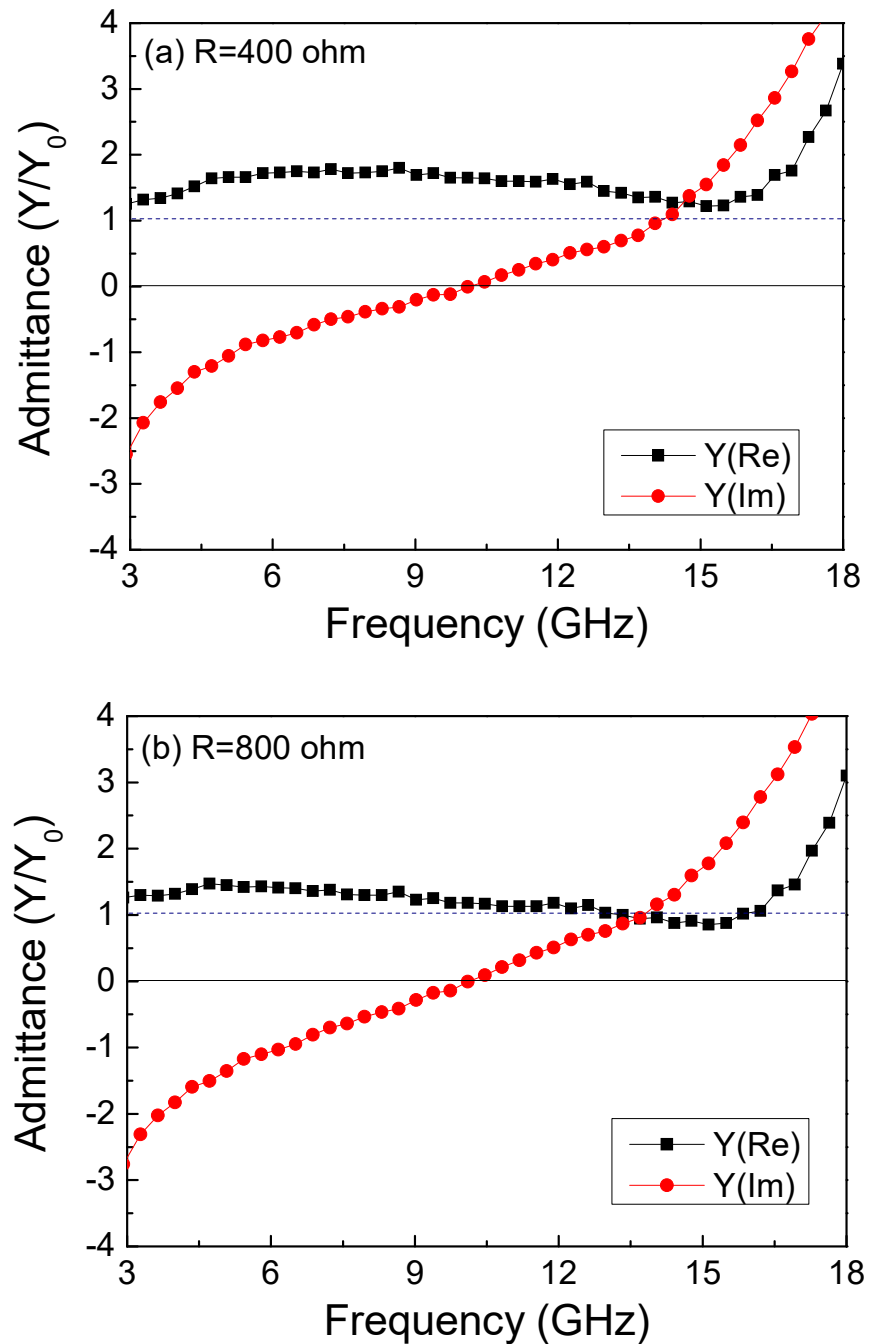

Fig. 7. Normalized real and imaginary parts of input admittance of SL-FSS with varying SL-FSS resistance: (a) $R=400 \Omega$, (b) $R=800 \Omega$

\section{Conclusions}

This work investigated the effect of an SL-FSS on the absorption properties of a grounded ferrite composite of CoZnW hexaferrite powders. It was verified by both HFSS simulation and circuit theory that microwave absorbing properties (reflection loss, bandwidth) were greatly improved by attaching a SL-FSS with controlled surface resistance onto grounded ferrite compos- ites. For the $S L-F S S$ with $R=800 \Omega$, the minimum reflection decreased to $-25 \mathrm{~dB}$ at $10 \mathrm{GHz}$ and the bandwidth was broadened to $7.5-12.5 \mathrm{GHz}$ with respect to $-10 \mathrm{~dB}$ reflection loss, which was significantly improved as compared with the grounded ferrite composite without FSS. This technique is efficient for a ferrite substrate with small magnetic loss where the real part of input admittance is small enough to satisfy the impedance matching. The attachment of a resistive FSS provides the additional real part of input admittance, leading to the impedance matching with free space.

\section{Acknowledgments}

This research was supported by Basic Science Research Program through the National Research Foundation of Korea (NRF) funded by the Ministry of Science, ICT \& Future Planning (grant number: 2016R1A2B4007970).

\section{REFERENCES}

[1] H.M. Musal Jr., H.T. Hahn, IEEE Trans, Magn. 25, 3851 (1989).

[2] S.S. Kim, S.B. Jo, K.I. Gueon, K.K. Choi, J.M. Kim, K.S. Kim, K.S. Chum, IEEE Trans. Magn. 27, 5462 (1991).

[3] W. Li, T. Wu, W. Wang, P. Zhai, J. Guan, J. Appl. Phys. 116, 044110 (2014).

[4] Z. Song, J. Xie, P. Zhou, X. Wang, T. Liu, L. Deng, J. Alloys Compd. 551, 677 (2013).

[5] Y. Liu, Y. Feng, X. Wu, X. Han, J. Alloys Compd. 520, 441 (2009).

[6] S. Sugimoto, K. Haga, T. Kagotani, K. Inomata, J. Magn. Magn. Mater. 290-291, 1188 (2005).

[7] A. Ghasemi, A. Hossienpour, A. Morisako, A. Saatchi, M. Salehi, J. Magn. Magn. Mater. 302, 429 (2006).

[8] F. Sakran, Y. Neve-Oz, IEEE Trans. Antenns Propag. 56, 2649 (2008).

[9] F. Arya, M. Matthew, H. Christian, V. Rudiger, IEEE Trans. Antennas Propag. 56, 340 (2008).

[10] F. Costa, A. Monorchio, G. Manara, IEEE Trans. Antennas Propag, 58, 1551 (2010).

[11] L. Sun, H. Cheng, Y. Zhou, J. Wang, Opt. Express 20, 4675 (2012).

[12] T. Liu, S.-S. Kim, Opt. Commnu. 117, 1401 (2016).

[13] R.J. Langley, E.A. Parker, Electronics Letters 18, 294 (1982). 